\title{
Probability sum rules and consistent quantum histories
}

\author{
Thomas F. Jordan* \\ Physics Department, University of Minnesota, Duluth, Minnesota 55812 USA \\ Eric D. Chisolm \\ Theoretical Division, Los Alamos National Laboratory, \\ Los Alamos, New Mexico 87545 USA
}

\begin{abstract}
An example shows that weak decoherence is more restrictive than the minimal logical decoherence structure that allows probabilities to be used consistently for quantum histories. The probabilities in the sum rules that define minimal decoherence are all calculated by using a projection operator to describe each possibility for the state at each time. Weak decoherence requires more sum rules. They bring in additional variables, that require different measurements and a different way to calculate probabilities, and raise questions of operational meaning. The example shows that extending the linearly positive probability formula from weak to minimal decoherence gives probabilities that are different from those calculated in the usual way using the Born and von Neumann rules and a projection operator at each time.
\end{abstract}

PACS numbers: 03.65.-w, 03.65.Yz

Keywords: decoherence, quantum histories, quantum trajectories

\section{INTRODUCTION}

A basic property of consistent quantum histories, whether they are used to construct an interpretation of quantum mechanics or to understand emergence of classical behavior, is that there are probability sum rules that describe decoherence; they give probabilities as sums of probabilities without quantum interference terms [1, 2, 3, 4, 5, 6, 7, 8, 9, 10]. The minimal logical decoherence structure for consistent quantum histories was described by

*email: tjordan@d.umn.edu

†email: echisolm@lanl.gov 
Omnès [2, 3, 8]. We will call it minimal decoherence. A different structure, which is easier to describe and use, is called weak decoherence [7]. It appears to be not completely appreciated that the two are different, that minimal decoherence does not imply weak decoherence as an outline of a proof too readily suggests [5], and that the sum rules that do imply weak decoherence [7] involve more far-reaching assumptions.

Here we give a simple example that makes this clear. The probabilities in the example satisfy the sum rules that define minimal decoherence but not those needed for weak decoherence. In minimal decoherence, each sum rule is for a probability, calculated using the Born and von Neumann rules, for the truth at successive times of propositions represented by projection operators. The sum rules of minimal decoherence relate all the probabilities that can be calculated this way, the probabilities for all possible outcomes of measurements that test those propositions, so minimal decoherence is consistent within itself. Weak decoherence requires more sum rules. It allows fewer histories. It brings in new variables that require new measurements and a new way to calculate probabilities. It involves more assumptions than are necessary. It raises questions of operational meaning. There is an altogether larger project [11, 12] we need not pursue. We will discuss this in the final section.

In the proposal of linearly positive histories and probabilities [13, 14], the foundation of the formula for probabilities is that it agrees with the probabilities calculated in the usual way for histories that satisfy the sum rules for weak decoherence [13]. Our example shows that this generally does not extend to histories that satisfy the sum rules for minimal decoherence. The linearly positive formula gives probabilities for histories in the example that are different from the probabilities calculated in the usual way using the Born and von Neumann rules and a projection operator at each time. Our perspective changes when we look beyond weak decoherence and bring minimal decoherence into view.

\section{HISTORIES AND PROBABILITIES}

We need to use only a simple set of quantum histories to make our point. Consider a history where at successive times a quantum system is in states represented by four different state vectors: first $|0\rangle$, then $|j\rangle$, then $|k\rangle$, and finally $|f\rangle$. Let $j \triangleright k \triangleright f$ denote this history. We let $|j\rangle$ be one of two orthonormal vectors $|1\rangle$ and $|2\rangle$, let $|k\rangle$ be one of three orthonormal vectors $|3\rangle,|4\rangle$ and $|5\rangle$, and let $|f\rangle$ be one of three orthonormal vectors $|6\rangle,|7\rangle$ and $|8\rangle$. In 
the set we consider, there will be no histories that go through $|3\rangle$ or $|4\rangle$ to $|8\rangle$ or through $|5\rangle$ to $|6\rangle$ or $|7\rangle$. Thus we consider ten $j \triangleright k \triangleright f$ histories:

$$
\begin{gathered}
1 \triangleright 3 \triangleright 6, \quad 1 \triangleright 4 \triangleright 6, \quad 2 \triangleright 3 \triangleright 6, \quad 2 \triangleright 4 \triangleright 6, \\
1 \triangleright 3 \triangleright 7, \quad 1 \triangleright 4 \triangleright 7, \quad 2 \triangleright 3 \triangleright 7, \quad 2 \triangleright 4 \triangleright 7, \\
1 \triangleright 5 \triangleright 8, \quad \text { and } \quad 2 \triangleright 5 \triangleright 8 .
\end{gathered}
$$

In the general formulation, there can be a different number of times, the number of possibilities at each time can be larger or smaller, the different possibilities at each time can be described by orthogonal projection operators for subspaces of states instead of by particular state vectors, the initial state can be mixed instead of pure, and all possible histories can be considered. Nothing we need is omitted in our simplification. The projection operators we use are simply formed from the state vectors.

The probability for the history $j \triangleright k \triangleright f$ is

$$
\langle f \mid k\rangle\langle k \mid j\rangle\langle j \mid 0\rangle\langle 0 \mid j\rangle\langle j \mid k\rangle\langle k \mid f\rangle \text {. }
$$

According to standard quantum mechanics, this is the probability, given the initial state represented by $|0\rangle$, for the truth at the successive times of the propositions represented by the projection operators $|j\rangle\langle j|| k\rangle,\langle k|$ and $|f\rangle\langle f|$. These projection operators are made to include the effect of the dynamics, as in the Heisenberg picture.

Different histories $j \triangleright k \triangleright f$ are independent, because at each time the different states are independent. If the system goes through one of these histories, there is no probability that it goes through another. Therefore, the probability that the result is one or the other of two of these histories, or of several, is the sum of the probabilities for those histories. When we say here that the system goes through either the history $j \triangleright 3 \triangleright f$ or the history $j \triangleright 4 \triangleright f$, for example, we mean to imply that it goes through either the state represented by $|3\rangle$ or the state represented by $|4\rangle$, not a state represented by a linear combination of $|3\rangle$ and $|4\rangle$.

When $|f\rangle$ is $|6\rangle$ or $|7\rangle$, we also consider

$$
\langle f|(|3\rangle\langle 3|+| 4\rangle\langle 4|)| j\rangle\langle j \mid 0\rangle\langle 0 \mid j\rangle\langle j|(|3\rangle\langle 3|+| 4\rangle\langle 4|)| f\rangle .
$$

This is the probability that the system goes from $|0\rangle$ to $|j\rangle$, and then to either $|3\rangle$ or $|4\rangle$ or a state represented by a vector in the subspace spanned by $|3\rangle$ and $|4\rangle$, and finally to $|f\rangle$. It is the probability, given the initial state represented by $|0\rangle$, for the truth at the successive 
times of the propositions represented by the projection operators $|j\rangle\langle j|| 3\rangle,\langle 3|+| 4\rangle\langle 4|$ and $|f\rangle\langle f|$. This can be when the system does not go through either the history $j \triangleright 3 \triangleright f$ or $j \triangleright 4 \triangleright f$. If we require that this probability (2.2) is just the sum of the probabilities for the histories $j \triangleright 3 \triangleright f$ and $j \triangleright 4 \triangleright f$, we conclude that

$$
\operatorname{Re}\langle f \mid 3\rangle\langle 3 \mid j\rangle\langle j \mid 0\rangle\langle 0 \mid j\rangle\langle j \mid 4\rangle\langle 4 \mid f\rangle=0
$$

for $j=1,2$ and $f=6,7$. For any $|f\rangle$, we consider the probability

$$
\langle f \mid k\rangle\langle k|(|1\rangle\langle 1|+| 2\rangle\langle 2|)| 0\rangle\langle 0|(|1\rangle\langle 1|+| 2\rangle\langle 2|)| k\rangle\langle k \mid f\rangle,
$$

given the initial state represented by $|0\rangle$, for the truth at the successive times of the propositions represented by the projection operators $|1\rangle\langle 1|+| 2\rangle\langle 2|| k\rangle,\langle k|$ and $|f\rangle\langle f|$. If we require that this is the sum of the probabilities for the histories $1 \triangleright k \triangleright f$ and $2 \triangleright k \triangleright f$, we conclude that

$$
\operatorname{Re}\langle f \mid k\rangle\langle k \mid 1\rangle\langle 1 \mid 0\rangle\langle 0 \mid 2\rangle\langle 2 \mid k\rangle\langle k \mid f\rangle=0
$$

for $k=3,4,5$ and $f=6,7,8$. When $|f\rangle$ is $|6\rangle$ or $|7\rangle$, we also consider the probability

$$
\langle f|(|3\rangle\langle 3|+| 4\rangle\langle 4|)(|1\rangle\langle 1|+| 2\rangle\langle 2|)| 0\rangle\langle 0|(|1\rangle\langle 1|+| 2\rangle\langle 2|)(|3\rangle\langle 3|+| 4\rangle\langle 4|)| f\rangle,
$$

given the initial state represented by $|0\rangle$, for the truth at the successive times of the propositions represented by the projection operators $|1\rangle\langle 1|+| 2\rangle\langle 2|| 3\rangle,\langle 3|+| 4\rangle\langle 4|$ and $|f\rangle\langle f|$. If we require that this is the sum of the probabilities for the four histories $1 \triangleright 3 \triangleright f, 1 \triangleright 4 \triangleright f$, $2 \triangleright 3 \triangleright f$, and $2 \triangleright 4 \triangleright f$, and assume that equations (2.3) and (2.5) hold, we conclude that

$$
\operatorname{Re}\langle f \mid 3\rangle\langle 3 \mid 1\rangle\langle 1 \mid 0\rangle\langle 0 \mid 2\rangle\langle 2 \mid 4\rangle\langle 4 \mid f\rangle+\operatorname{Re}\langle f \mid 4\rangle\langle 4 \mid 1\rangle\langle 1 \mid 0\rangle\langle 0 \mid 2\rangle\langle 2 \mid 3\rangle\langle 3 \mid f\rangle=0
$$

for $f=6,7$. These sum rules generally do not hold. The probabilities (2.2), (2.4) and (2.6) generally are not the same as the sums of the probabilities for the individual histories, because the quantum interference terms (2.3), (2.5), and (2.7) generally are not zero. The condition that the interference terms are zero, so that the sum rules do hold, is called decoherence. These sum rules relate probabilities calculated by using a projection operator to describe each possibility for the state at each time. Sums of probabilities correspond to sums of projection operators. We will call these projection sum rules. They ensure consistency among these probabilities. They define the minimal logical structure of decoherence with this consistency. We will call it minimal decoherence. It was described by Omnès [2, 3, 8]. 
Weak decoherence [7] is the condition that

$$
\operatorname{Re}\langle f \mid k\rangle\langle k \mid j\rangle\langle j \mid 0\rangle\left\langle 0 \mid j^{\prime}\right\rangle\left\langle j^{\prime} \mid k^{\prime}\right\rangle\left\langle k^{\prime} \mid f\right\rangle=0 \text { unless } j=j^{\prime} \text { and } k=k^{\prime} .
$$

For the histories we are considering, this means that Eqs. (2.3), (2.5), and (2.7) hold and that each of the two terms of Eqs. (2.7) is separately zero. Our example will show that the latter is not implied by projection sum rules. Minimal decoherence does not imply weak decoherence. A proof that the two terms of Eqs. (2.7) are separately zero can be made [7] with an additional assumption: either that

$$
\langle f|(|3\rangle\langle 3 \mid 1\rangle\langle 1|+| 4\rangle\langle 4 \mid 2\rangle\langle 2|)| 0\rangle\langle 0|(|1\rangle\langle 1 \mid 3\rangle\langle 3|+| 2\rangle\langle 2 \mid 4\rangle\langle 4|)| f\rangle
$$

is the sum of the probabilities for the histories $1 \triangleright 3 \triangleright f$ and $2 \triangleright 4 \triangleright f$, or that

$$
\langle f|(|4\rangle\langle 4 \mid 1\rangle\langle 1|+| 3\rangle\langle 3 \mid 2\rangle\langle 2|)| 0\rangle\langle 0|(|1\rangle\langle 1 \mid 4\rangle\langle 4|+| 2\rangle\langle 2 \mid 3\rangle\langle 3|)| f\rangle
$$

is the sum of the probabilities for the histories $1 \triangleright 4 \triangleright f$ and $2 \triangleright 3 \triangleright f$. These are not projection sum rules. They are different. We will call them history sum rules. They include quantities, like (2.9) and (2.10), that are not calculated by using projection operators to describe the possibilities for the state at each time. Our example will show that history sum rules are not implied by projection sum rules. They are an additional assumption.

These additional sum rules distinguish weak from minimal decoherence. They introduce a new kind of history, which cannot be described with a proposition represented by a projection operator at each time. Mathematically, they are an inviting generalization, using

operators to represent histories and sums of operators for compositions of histories [7], but the corresponding operational procedures are not at all clear. We will consider this further in the final section, but first we describe our example.

\section{EXAMPLE}

Our example is specified by the overlaps of its state vectors. Let

$$
\begin{aligned}
|0\rangle & =\frac{1}{\sqrt{2}}|1\rangle+\frac{i}{\sqrt{2}}|2\rangle \\
|1\rangle & =\frac{1}{2}|3\rangle+\frac{1}{2}|4\rangle+\frac{1}{\sqrt{2}}|5\rangle \\
|2\rangle & =\frac{1}{2}|3\rangle+\frac{1}{2}|4\rangle-\frac{1}{\sqrt{2}}|5\rangle
\end{aligned}
$$




$$
\begin{aligned}
|6\rangle & =\frac{1-i}{2}|3\rangle+\frac{1+i}{2}|4\rangle \\
|7\rangle & =\frac{1+i}{2}|3\rangle+\frac{1-i}{2}|4\rangle \\
|8\rangle & =|5\rangle .
\end{aligned}
$$

It is easy to see that Eqs. (2.3), (2.5), and (2.7) are satisfied. The two terms of Eqs. (2.7) are not separately zero. They are

$$
\begin{aligned}
& \langle 6 \mid 3\rangle\langle 3 \mid 1\rangle\langle 1 \mid 0\rangle\langle 0 \mid 2\rangle\langle 2 \mid 4\rangle\langle 4 \mid 6\rangle=1 / 16 \\
& \langle 6 \mid 4\rangle\langle 4 \mid 1\rangle\langle 1 \mid 0\rangle\langle 0 \mid 2\rangle\langle 2 \mid 3\rangle\langle 3 \mid 6\rangle=-1 / 16 \\
& \langle 7 \mid 3\rangle\langle 3 \mid 1\rangle\langle 1 \mid 0\rangle\langle 0 \mid 2\rangle\langle 2 \mid 4\rangle\langle 4 \mid 7\rangle=-1 / 16 \\
& \langle 7 \mid 4\rangle\langle 4 \mid 1\rangle\langle 1 \mid 0\rangle\langle 0 \mid 2\rangle\langle 2 \mid 3\rangle\langle 3 \mid 7\rangle=1 / 16 .
\end{aligned}
$$

The projection sum rules are satisfied. The history sum rules are not.

The probability (2.1) is $1 / 16$ for each of the four histories $j \triangleright k \triangleright 6$ and $1 / 16$ for each of the four histories $j \triangleright k \triangleright 7$; it is $1 / 4$ for each of the two histories $j \triangleright k \triangleright 8$. The probability that a history goes to a particular $|f\rangle$ through $|1\rangle$ is the same as the probability that it goes there through $|2\rangle$, and the probability that a history goes to a particular $|f\rangle$ through $|3\rangle$ is the same as the probability that it goes there through $|4\rangle$. The probability that a history goes to $|6\rangle$ is $1 / 4$, the probability that a history goes to $|7\rangle$ is $1 / 4$, and the probability that a history goes to $|8\rangle$ is $1 / 2$. From Eqs. (3.1) we find that

$$
|0\rangle=\frac{1+i}{2 \sqrt{2}}|3\rangle+\frac{1+i}{2 \sqrt{2}}|4\rangle+\frac{1-i}{2}|5\rangle
$$

and can see that

$$
\begin{aligned}
& \langle 6 \mid 0\rangle\langle 0 \mid 6\rangle=1 / 4 \\
& \langle 7 \mid 0\rangle\langle 0 \mid 7\rangle=1 / 4 \\
& \langle 8 \mid 0\rangle\langle 0 \mid 8\rangle=1 / 2 .
\end{aligned}
$$

The probabilities add up. Nothing has been left out. The vector $|0\rangle$ is in the subspace spanned by $|1\rangle$ and $|2\rangle$. A vector that would make a complete set of three orthonormal vectors with $|1\rangle$ and $|2\rangle$ would be orthogonal to $|0\rangle$, so it could not change anything we have considered.

In terms of probabilities, we can see directly that the history sum rules are not satisfied. From Eqs. (3.2) and the probability 1/16 for each of the histories $j \triangleright k \triangleright 6$ and $j \triangleright k \triangleright 7$, we 
see that the quantity (2.9) is $1 / 4$ when $|f\rangle$ is $|6\rangle$ and zero when $|f\rangle$ is $|7\rangle$, and the quantity (2.10) is zero when $|f\rangle$ is $|6\rangle$ and $1 / 4$ when $|f\rangle$ is $|7\rangle$. The quantity (2.9) is not the sum of the probabilities for the histories $1 \triangleright 3 \triangleright f$ and $2 \triangleright 4 \triangleright f$, and the quantity (2.10) is not the sum of the probabilities for the histories $1 \triangleright 4 \triangleright f$ and $2 \triangleright 3 \triangleright f$. These sums are both $1 / 8$.

We can also see directly that the projection sum rules do not imply the history sum rules. The values of the quantities (2.9) and (2.10) are changed when $|6\rangle$ and $|7\rangle$ are interchanged. To check that the projection sum rules are satisfied, we looked at the values of all the quantities in them. We can see that none of these values are changed when $|6\rangle$ and $|7\rangle$ are interchanged. This confirms that the quantities (2.9) and (2.10) in the history sum rules are not determined by measurements of quantities in the projection sum rules.

\section{LINEARLY POSITIVE HISTORIES AND PROBABILITIES}

To consider the proposal of linearly positive histories and probabilities [13, 14], we look at

$$
p(j \triangleright k \triangleright f)=\operatorname{Re}\langle 0 \mid f\rangle\langle f \mid k\rangle\langle k \mid j\rangle\langle j \mid 0\rangle .
$$

The proposal assumes that if $p(j \triangleright k \triangleright f)$ is not negative, it is the probability for the history $j \triangleright k \triangleright f$. For a set of histories that satisfies the conditions (2.8) for weak decoherence, $p(j \triangleright k \triangleright f)$ is nonnegative for every history $j \triangleright k \triangleright f$ in the set and is the same as the probability (2.1) calculated in the usual way [13]. This is generally not true for a set of histories that satisfies the conditions for minimal decoherence. For the histories in our example, we find that $p(j \triangleright k \triangleright f)$ is $1 / 8$ for each of the four histories $1 \triangleright 3 \triangleright 6,2 \triangleright 4 \triangleright 6,2 \triangleright 3 \triangleright 7$, $1 \triangleright 4 \triangleright 7$ and is zero for each of the four histories $2 \triangleright 3 \triangleright 6,1 \triangleright 4 \triangleright 6,1 \triangleright 3 \triangleright 7,2 \triangleright 4 \triangleright 7$. The probability (2.1) is $1 / 16$ for each of these eight histories. For each of the two histories $1 \triangleright 5 \triangleright 8$ and $2 \triangleright 5 \triangleright 8$, we find that $p(j \triangleright k \triangleright f)$ is $1 / 4$, which is the same as the probability (2.1). The proposal provides a simple formula for probabilities for weakly decoherent histories, but it is not a reliable extension to other histories.

\section{TRAJECTORY GRAPHS}

Trajectory graphs [6, 15] can illustrate the difference between minimal and weak decoherence. The trajectory graph for our example of minimal decoherence is shown in Fig. 11. 
There is a column for each time. The different points in the column represent the different states the system can be in at that time in its history. There is a path across the graph for each history $j \triangleright k \triangleright f$. These paths are also called trajectories.

Weak decoherence allows two states at different times to be connected by at most two different histories [15]. For the numbers of states in our example, it allows at most two different histories $j \triangleright k \triangleright f$ and $j^{\prime} \triangleright k^{\prime} \triangleright f$ between $|0\rangle$ and a particular $|f\rangle$. This is because the condition (2.8) for weak decoherence requires that the phases of $\langle 0 \mid j\rangle\langle j \mid k\rangle\langle k \mid f\rangle$ and $\left\langle 0 \mid j^{\prime}\right\rangle\left\langle j^{\prime} \mid k^{\prime}\right\rangle\left\langle k^{\prime} \mid f\right\rangle$ differ by $\pi / 2$, and there can be at most two different complex numbers with phases that differ by $\pi / 2$. Trajectory graphs allowed by weak decoherence for the numbers of states in our example are shown in Fig. 2.

Here is a consequence of weak decoherence [15] that is also implied by minimal decoherence.

Theorem: Projection sum rules imply that a state $|j\rangle$ does not occur at two different times if the histories between those times go through a set of states that does not contain $|j\rangle$.

Proof: The probability for that is the sum of the probabilities for all the histories that go through $|j\rangle$ at those two times, which becomes

$$
\begin{gathered}
\sum_{k}\langle f|. .1 . .| j\rangle\langle j|. .1 . .| k\rangle\langle k|. .1 . .| j\rangle\langle j|. .1 . .| 0\rangle\langle 0|. .1 . .| j\rangle\langle j|. .1 . .| k\rangle\langle k|. .1 . .| j\rangle\langle j|. .1 . .| f\rangle \\
=\sum_{k}\langle f \mid j\rangle\langle j \mid k\rangle\langle k \mid j\rangle\langle j \mid 0\rangle\langle 0 \mid j\rangle\langle j \mid k\rangle\langle k \mid j\rangle\langle j \mid f\rangle
\end{gathered}
$$

when projection sum rules replace the sum over probabilities with the sum over projection operators

$$
\sum_{m}|m\rangle\langle m|=1
$$

inserted left and right, for each set of states except the two sets that contain $|j\rangle$ and one set of states $|k\rangle$ in between that does not contain $|j\rangle$ but contains at least two states $|k\rangle$ and $\left|k^{\prime}\right\rangle$ on histories that go through $|j\rangle$ at both times. The result is zero because the projection sum rules imply that

$$
\operatorname{Re}\langle f \mid j\rangle\langle j \mid k\rangle\langle k \mid j\rangle\langle j \mid 0\rangle\langle 0 \mid j\rangle\left\langle j \mid k^{\prime}\right\rangle\left\langle k^{\prime} \mid j\right\rangle\langle j \mid f\rangle=0
$$

when $|k\rangle$ and $\left|k^{\prime}\right\rangle$ are different. This completes the proof of the theorem.

In particular, this does not allow cyclic histories where there is change. 


\section{DISCUSSION}

Does it matter that there is a difference between minimal and weak decoherence? Actual decoherence that is produced physically is more likely to be medium decoherence [7], the condition that

$$
\langle f \mid k\rangle\langle k \mid j\rangle\langle j \mid 0\rangle\left\langle 0 \mid j^{\prime}\right\rangle\left\langle j^{\prime} \mid k^{\prime}\right\rangle\left\langle k^{\prime} \mid f\right\rangle=0 \text { unless } j=j^{\prime} \text { and } k=k^{\prime},
$$

which is more restrictive than either minimal or weak decoherence. It is more likely that physical processes will reduce magnitudes to zero than that they will line up phases to satisfy equations involving real parts. In the important application of consistent histories to the description of a measuring process in fully quantum terms, the histories are shown to satisfy medium decoherence [16].

It is for logical reasons that we want to know the minimum decoherence structure needed for consistent use of probabilities. Indeed, Omnès has said that

when it comes to proving beyond any doubt that a statement is meaningless whatever its context, ... saying for instance through which arm of an interferometer a photon goes ... one must make sure that no possibility has been left out, and one must use necessary and sufficient conditions. [4, page 346]

Griffiths has expressed different interests, which have not made it a priority to pin down the necessary and sufficient conditions:

The use of a weak consistency condition has the advantage that it allows a wider class of consistent families in the quantum formalism. However, greater generality is not always a virtue in theoretical physics, and it remains to be seen whether there are "realistic" physical situations where it is actually helpful to employ weak rather than strong consistency. [9, following Eq. (3.16)]

Even weaker conditions may work in certain cases. The subject has not been exhaustively studied. However, the [stronger, essentially medium decoherence] conditions are easier to apply in actual calculations than are any of the weaker conditions, and seem adequate to cover all situations of physical interest which have been studied up till now. Consequently, we shall refer to them from now on 
as "the consistency conditions", while leaving open the possibility that further study may indicate the need to use a weaker condition that enlarges the class of consistent families. [10, page 142]

There has not been established recognition of what the necessary and sufficient conditions are.

We have three different logical structures: minimal, weak, and medium decoherence. Each is well defined and consistent within itself. Minimal decoherence is all that is necessary. Its requirements are minimal. It imposes the weakest restrictions and includes the most histories. It includes all histories that satisfy projection sum rules. The probabilities in projection sum rules are calculated by using the Born rule and the von Neumann rule, the most clearly established rules for the use of probability in quantum mechanics. Their use in minimal decoherence is always to calculate a probability for the truth at successive times of propositions represented by projection operators, for example the probability (2.1), (2.2), (2.4), or (2.6). Projection sum rules relate all the probabilities that can be calculated that way, the probabilities for all possible outcomes of measurements made to test those propositions, so there is consistency. History sum rules contain quantities that are taken to be probabilities but can not be calculated that way, for example the quantities (2.9) and (2.10). The operational meaning of these quantities is not clear to us. They can not be determined by measurements made to test a proposition represented by a projection operator at each time. Weak decoherence requires history sum rules. It allows fewer histories, and it brings in new variables that require new measurements and a new way to calculate probabilities. It is more than is necessary.

Fortunately, most logical arguments involving weak decoherence use only a part of it that is included also in minimal decoherence, the conditions for histories that differ at only one time, like Eqs. (2.3) and (2.5). This is all that was originally considered [1]. Minimal decoherence implies more; for example, it implies Eq. (2.7).

There is an argument for going in the other direction, from weak to medium decoherence, rather than from weak to minimal: if weak decoherence is assumed to apply in a universal manner to systems and composites of separate and independent systems, it implies medium decoherence [17]. We see no reason to make this assumption, because we see that weak decoherence is not necessary; minimal decoherence is enough. If we begin with minimal 
instead of weak decoherence, there is no argument that gives medium decoherence like the argument using composite systems does from weak decoherence, no argument that bypasses the additional assumptions needed for weak decoherence. Our example illustrates the difference between minimal decoherence and weak decoherence. We discuss the assumptions needed for these. We do not consider stronger assumptions that imply a stronger form of decoherence which may be suitable for various purposes.

Clarifications we have achieved highlight questions that remain open. Can quantum histories be defined broadly enough to be the elements of a sample space with a probability measure in the sense of the axioms of probability theory stated by Kolmogorov [18]? Kolmogorov requires that any two disjoint elements of the sample space can be joined by an "or" operation. Can that be done simply by adding operators? For an array of states numbered as in our example, the operators $|f\rangle\langle f \mid 3\rangle\langle 3 \mid j\rangle\langle j|$ and $|f\rangle\langle f \mid 4\rangle\langle 4 \mid j\rangle\langle j|$ correspond to the histories $j \triangleright 3 \triangleright f$ and $j \triangleright 4 \triangleright f$ and can be used as descriptions of these histories. The sum of these operators,

$$
|f\rangle\langle f \mid 3\rangle\langle 3 \mid j\rangle\langle j|+| f\rangle\langle f \mid 4\rangle\langle 4 \mid j\rangle\langle j|=| f\rangle\langle f|(|3\rangle\langle 3|+| 4\rangle\langle 4|)| j\rangle\langle j|
$$

is used in Eq. (2.2). It describes the history specified by propositions at successive times represented by the projection operators $|j\rangle\langle j|| 3\rangle,\langle 3|+| 4\rangle\langle 4|$ and $|f\rangle\langle f|$, for which Eq. (2.2) is the probability. We can take this history to be the "or" composition of the histories $j \triangleright 3 \triangleright f$ and $j \triangleright 4 \triangleright f$. What is the "or" composition of the histories $1 \triangleright 3 \triangleright f$ and $2 \triangleright 4 \triangleright f$ ? Does the operator

$$
|f\rangle\langle f \mid 3\rangle\langle 3 \mid 1\rangle\langle 1|+| f\rangle\langle f \mid 4\rangle\langle 4 \mid 2\rangle\langle 2|=| f\rangle\langle f|(|3\rangle\langle 3 \mid 1\rangle\langle 1|+| 4\rangle\langle 4 \mid 2\rangle\langle 2|)
$$

describe a history? It does not describe a history with a proposition represented by projection operator at each time. If there is any "or" composition of the histories $1 \triangleright 3 \triangleright f$ and $2 \triangleright 4 \triangleright f$, described by this sum of operators or otherwise, how would measurements affirm it? How could the probability for it be understood?

For the "or" composition of the histories $j \triangleright 3 \triangleright f$ and $j \triangleright 4 \triangleright f$, described by the operator sum (6.2), we have the probability (2.2) for the truth at successive times of the propositions represented by the projection operators $|j\rangle\langle j|| 3\rangle,\langle 3|+| 4\rangle\langle 4|$ and $|f\rangle\langle f|$. This kind of probability for a sequence of events was first discussed in the literature by Wigner [19] and by Aharanov, Bergmann, and Lebowitz [20]. Sometimes its form is imagined to come 
from successive "collapses of the wave function," but it can be understood simply as the probability for a sequence of measurements to have a certain result, without any reference to wave function collapse [21, 22]. Its operational meaning is clear. Could there be a more general probability formula that applies to the "or" composition of the histories $1 \triangleright 3 \triangleright f$ and $2 \triangleright 4 \triangleright f$ as well? What would the measurement procedure for it be? How could experiments verify that it gives the right answer?

A tensor-product enlargement of the mathematical structure has been proposed that allows a projection operator for every proposition that is to have a probability [11, 12]. It is hoped that this may provide a foundation for a quantum theory of gravity. It has not answered the questions of operational meaning. Measurements that could test the propositions represented by all the new projection operators have not been described. We think that for ordinary quantum mechanics it may be more reasonable to just use minimal decoherence. We feel less need to find meaning for all the sum rules of weak decoherence when we consider that minimal decoherence does not require weak decoherence and that minimal decoherence provides a framework for consistent use of probabilities for all the possibilities described by a sequence of propositions represented by projection operators at different times, the probabilities for all the possible results of measurements that test those propositions.

[1] R. B. Griffiths, Journal of Statistical Physics 36, 219 (1984).

[2] R. Omnès, Journal of Statistical Physics 53, 893 (1988).

[3] R. Omnès, Ann. Phys. (N.Y.) 201, 354 (1990).

[4] R. Omnès, Rev. Mod. Phys. 64, 339 (1992).

[5] H. F. Dowker and J. J. Halliwell, Phys. Rev. D 46, 1580 (1992).

[6] R. B. Griffiths, Phys. Rev. Lett. 70, 2201 (1993).

[7] M. Gell-Mann and J. B. Hartle, Phys. Rev. D 47, 3345 (1993).

[8] R. Omnès, The Interpretation of Quantum Mechanics (Princeton University Press, Princeton, N. J., 1994), particularly Chapter 4, Appendix A.

[9] R. B. Griffiths, Phys. Rev. A 54, 2759 (1996).

[10] R. B. Griffiths, Consistent Quantum Theory (Cambridge University Press, Cambridge, U. K., 
2002), particularly Chapter 10.

[11] C. J. Isham, J. Math. Phys. 35, 2157 (1994).

[12] C. J. Isham and N. Linden, J. Math. Phys. 35, 5452 (1994).

[13] S. Goldstein and D. N. Page, Phys. Rev. Lett. 74, 3715 (1995).

[14] J. B. Hartle, Phys. Rev. A 70, 02210 (2004).

[15] E. Chisolm, E. C. G. Sudarshan, and T. F. Jordan, Intern. J. Theor. Phys. 35, 485 (1996).

[16] R. B. Griffiths, reference [10], particularly Chapters 17 and 18.

[17] L. Diósi, Phys. Rev. Lett. 92, 170401 (2004).

[18] A. N. Kolmogorov, Foundations of the Theory of Probability (Chelsea Publishing Company, New York, N.Y., 1950).

[19] E. P. Wigner, Am. J. Phys. 31, 6 (1963).

[20] Y. Aharanov, P. G. Bergmann, and J. L. Lebowitz, Phys. Rev. 134, B1410 (1964).

[21] L. E. Ballentine, Quantum Mechanics: A Modern Development (World Scientific, Singapore, 1998), particularly Section 9.6.

[22] R. B. Griffiths, reference [10], particularly Section 18.2. 


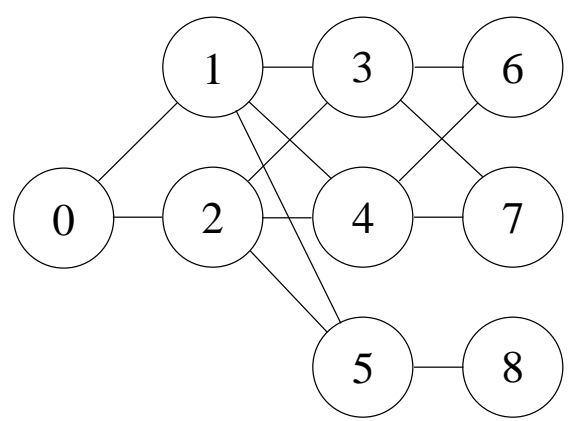

FIG. 1: The trajectory graph representing the set of histories in our example.
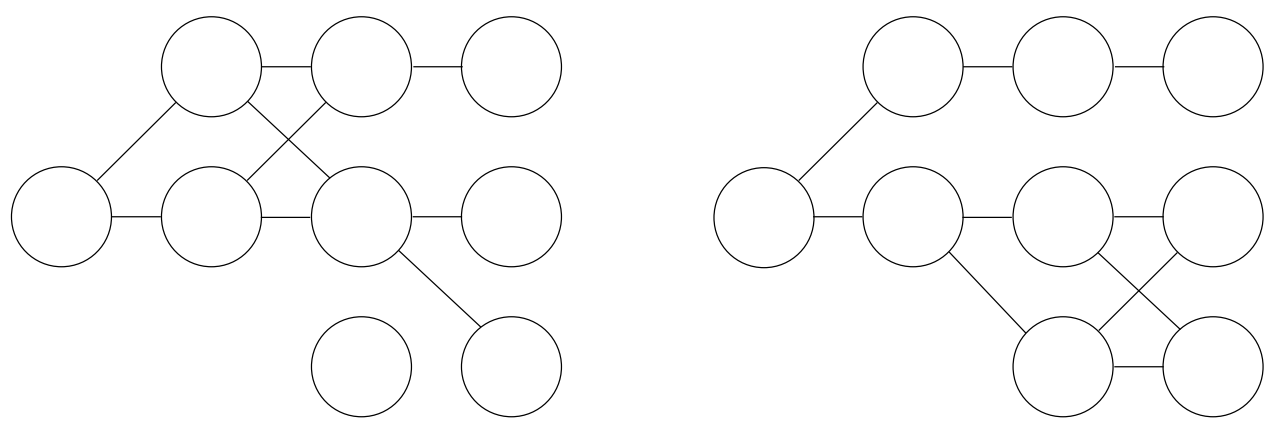

FIG. 2: Trajectory graphs allowed by weak decoherence. 